\title{
Comparative Evaluation of Two Venous Sampling Techniques for the Assessment of Pancreatic Insulin and Zinc Release upon Glucose Challenge
}

\author{
Anil Kumar Pillai, ${ }^{1}$ William Silvers, ${ }^{1}$ Preston Christensen, ${ }^{1}$ Matthew Riegel, ${ }^{2}$ \\ Beverley Adams-Huet, ${ }^{3}$ Ildiko Lingvay, ${ }^{3}$ Xiankai Sun, ${ }^{1,4}$ and Orhan K. Öz ${ }^{1}$ \\ ${ }^{1}$ Department of Radiology, University of Texas Southwestern Medical Center, Dallas, TX 75390, USA \\ ${ }^{2}$ Animal Resources Center, University of Texas Southwestern Medical Center, Dallas, TX 75390, USA \\ ${ }^{3}$ Departments of Internal Medicine and Clinical Sciences, University of Texas Southwestern Medical Center, Dallas, TX 75390, USA \\ ${ }^{4}$ Advanced Imaging Research Center, University of Texas Southwestern Medical Center, Dallas, TX 75390, USA \\ Correspondence should be addressed to Anil Kumar Pillai; anil.pillai@utsouthwestern.edu
}

Received 19 April 2015; Revised 11 July 2015; Accepted 12 July 2015

Academic Editor: Hiroshi Okamoto

Copyright (C) 2015 Anil Kumar Pillai et al. This is an open access article distributed under the Creative Commons Attribution License, which permits unrestricted use, distribution, and reproduction in any medium, provided the original work is properly cited.

\begin{abstract}
Advances in noninvasive imaging modalities have provided opportunities to study $\beta$ cell function through imaging zinc release from insulin secreting $\beta$ cells. Understanding the temporal secretory pattern of insulin and zinc corelease after a glucose challenge is essential for proper timing of administration of zinc sensing probes. Portal venous sampling is an essential part of pharmacological and nutritional studies in animal models. The purpose of this study was to compare two different percutaneous image-guided techniques: transhepatic ultrasound guided portal vein access and transsplenic fluoroscopy guided splenic vein access for ease of access, safety, and evaluation of temporal kinetics of insulin and zinc release into the venous effluent from the pancreas. Both techniques were safe, reproducible, and easy to perform. The mean time required to obtain desired catheter position for venous sampling was 15 minutes shorter using the transsplenic technique. A clear biphasic insulin release profile was observed in both techniques. Statistically higher insulin concentration but similar zinc release after a glucose challenge was observed from splenic vein samples, as compared to the ones from the portal vein. To our knowledge, this is the first report of percutaneous methods to assess zinc release kinetics from the porcine pancreas.
\end{abstract}

\section{Introduction}

In 2013, the prevalence of diabetes mellitus was 382 million people worldwide and is expected to rise to 592 million by 2035, an increase of 55\% [1]. Approximately 37 million persons in the United States have diabetes, of which type 2 accounts for the vast majority (90\%) of cases. According to the Centers for Disease Control and Prevention, diabetes mellitus is the 7th leading cause of mortality and contributes to $38 \%$ of deaths before the age of 60 in the United States [2]. Early detection and lifestyle changes can have a positive impact in preventing complications of diabetes.

Advances in noninvasive imaging modalities have provided opportunities to study the pancreatic $\beta$ cell function through imaging zinc release from $\beta$ cells, as it is coreleased with insulin $[3,4]$. The highest concentration of zinc in the body has been detected in the pancreatic $\beta$ cells within the Islets of Langerhans, which is crucial for the synthesis, secretion, and signaling of insulin [5-7]. Alterations to zinc transporters on $\beta$ cells have been linked to altered insulin synthesis, secretion, and increased risk of diabetes [8-10]. The insulin response to a rapid intravenous glucose challenge has been reported to be biphasic, where the first phase results from the release of preformed insulin granules [11, 12]. The subsequent phase, which is more sustained, is due to mobilization of a stored granule pool. During insulin secretion, zinc is coreleased into the extracellular islet space [13]. The temporal dynamics of zinc release from $\beta$ cells 
have been studied at the cellular level using isolated islets [1], but little is known about the dynamics of zinc release in vivo. Imaging of zinc release as a surrogate marker for insulin secretion is the basis for developing imaging probes to noninvasively monitor $\beta$ cell function [3]. Understanding the temporal secretory pattern of zinc and insulin corelease after a glucose challenge is essential for proper timing of probe administration. Continuous peripancreatic venous sampling after glucose challenge provides an accurate temporal profile of zinc release, whereby the biphasic response could be assessed.

Animal models serve as an important research tool for nutritional physiological studies, in which swine are commonly used owing to the numerous anatomical and physiological similarities between porcine and human [14]. Traditionally, portal vein sampling in swine involved a surgical procedure including an abdominal incision and placement of a vascular access port for repeated sampling $[15,16]$. Herein, we describe two different percutaneous techniques to perform blood sampling from the portal and splenic veins and compare the temporal sequence of insulin and zinc release after a glucose challenge in each technique.

\section{Materials and Methods}

All experimental procedures involving animals were approved by the UT southwestern institutional animal care and use committee (IACUC). The procedure was performed on six adult Ossabaw pigs weighing between 80 and 260 pounds under general anesthesia [17].

2.1. CT Angiography. CT angiography of the first pig was done to evaluate the normal portal anatomy for planning the portal venous access. The images were acquired on a Siemens Biograph PET/CT scanner equipped with a 64slice CT scanner (Siemens Healthcare USA, Inc., Malvern, PA). The right femoral vein was accessed under ultrasound guidance. Over a guidewire, a 5-French straight flush catheter was advanced and the tip positioned in the mid caudal vena cava. Catheter position was confirmed on the scout scans. A contrast agent (Omnipaque 350, GE Health care, Princeton, $\mathrm{NJ}$ ) was injected at $3 \mathrm{~mL} / \mathrm{sec}$ for a total volume of $154 \mathrm{~mL}$ $(2 \mathrm{~mL} / \mathrm{kg})$. Image acquisition was performed at 18 seconds for the arterial phase and 29 seconds for the portal venous phase [18]. Images were reconstructed using a Siemens clinical workstation in the TrueD CT software environment.

2.2. Venous Sampling Techniques. The portal vein sampling was done in a dedicated fluoroscopy animal suite (Infinix AX, Toshiba Inc., Tokyo, Japan). Ultrasound guided saphenous venous access for blood draws and glucose infusion was obtained prior to the procedure. Two different techniques were used for portal venous sampling as described below.

2.2.1. Technique 1: Transhepatic Ultrasound Guided Portal Vein Access. Each of the three pigs sampled by this technique was positioned on the fluoroscopic table on the left lateral decubitus position. The right upper abdomen was shaved and cleaned. Preprocedure ultrasound scan using a $3.5 \mathrm{mHz}$ curvilinear transducer (Siemens Elegra, Erlangen, Germany) was performed to identify a suitable portal vein branch (right, left, or main portal vein). Using an intercostal approach, a 21-gauge needle was advanced under real time visualization into the targeted portal vein. Two methods were used to visualize the needle while performing the ultrasound guided access: in-plane (long axis) and out-of-plane (short axis). The former follows the tip of the needle, which was seen as a bright spot. As the tip was being advanced, the probe was angulated in the direction of the needle tip in small increments until the tip was visualized within the target. The latter visualizes the entire needle. The needle was kept in the same plane as the ultrasound beam. By adjusting the needle to be in-plane with the ultrasound beam while keeping the target in the field of view, the needle was advanced into the target. After fluoroscopic confirmation of the access by injecting 5-10 $\mathrm{mL}$ of Omnipaque 350 , a 0.018 inch wire was passed into a branch of the right or left portal vein, over which a 5-French $(\mathrm{F})$ triaxial introducer (Neff set, Cook, Bloomington, IN) was advanced to establish the access. Procedure time was defined as the duration from the initial needle stick as recorded by the anesthesia team in their procedure log to the time stamp on the fluoroscopic image confirming the venous access. Through the introducer sheath, a $4 \mathrm{~F}$ angled catheter was advanced for blood sampling.

\subsubsection{Technique 2: Transsplenic Fluoroscopy Guided Splenic} Vein Access. Each of the three pigs sampled by this technique was positioned on the fluoroscopic table in the left lateral decubitus position. The left upper abdomen was shaved and cleaned. Preprocedure ultrasound scan using a $3.5 \mathrm{mHz}$ curvilinear transducer (Siemens Elegra, Erlangen, Germany) was performed to identify the spleen. Using an intercostal approach, a 21-gauge needle was advanced under real time visualization into the splenic parenchyma. Omnipaque 350 (5-10 mL) was gently injected into the splenic parenchyma under real time fluoroscopy until the splenic vein was opacified (Figure 3(a)). A second 21-gauge needle was advanced into the splenic vein under fluoroscopic guidance using a triangulation technique [19]. The C arm was moved back and forth between 2 orthogonal positions: 1 parallel and 1 oblique to the line of puncture. This orients the operator if the needle is off plane in the mediolateral (parallel) or craniocaudal (oblique) plane. Adjustments can then be made in several small steps until the needle hits the target (Figure 3(b)). Once the needle was visualized within the targeted splenic vein, $5-10 \mathrm{~mL}$ of Omnipaque 350 was injected and a venogram performed to confirm access. A 0.018 -inch wire was passed into the splenic vein, over which a $5 \mathrm{~F}$ triaxial introducer (Neff set, Cook, Bloomington, IN) was advanced to maintain the access. Procedure time was defined as the duration from the initial needle stick as recorded by the anesthesia team in their procedure log to the time stamp on the fluoroscopic image confirming the venous access. Sampling was done through this sheath. 
2.3. Measurement of Zinc and Insulin Concentration. After venous access was established, $2 \mathrm{~mL}$ of blood was obtained from the portal/splenic vein for baseline measurements. A dextrose bolus $(0.3 \mathrm{~g} / \mathrm{kg})$ was administered within 20 seconds into the saphenous vein. The first sample was obtained $2 \mathrm{~min}$ after the dextrose injection. Sampling was subsequently performed every $2 \mathrm{~min}$ for a total of 30 samples. Plasma samples were prepared from the blood samples for the measurement of zinc and insulin concentrations. Plasma zinc concentration was measured using an inductively coupled plasma mass spectrometer (ICP-MS, Agilent 7700, Santa Clara, CA, USA). Zinc samples were prepared by diluting $100 \mu \mathrm{L}$ of plasma into $900 \mu \mathrm{L}$ of $10 \%$ hydrochloric acid (HCL) (trace metal, $3.6 \mathrm{ppb} \mathrm{Zn}$ ) in nanopure water (Millipore Gradient MilliQ water system, Billerica, MA). Samples were vortexed to mix thoroughly and centrifuged at $14,000 \mathrm{rpm}$ for $10 \mathrm{~min}$ to remove lipid and protein pellets. The results were determined using a calibration curve generated from a commercially available zinc standard (Inorganic Ventures, Christiansburg, VA USA). Insulin concentration in the plasma samples was measured using commercially available porcine insulin ELISA kits (Alpco).

2.4. Statistics. The variables evaluated in the two groups were ease of access as measured by the time to establish access (catheter positioned in desired location) and concentration of insulin and zinc in the plasma samples. The ease of access was determined using the average time to establish access after the start of the procedure. Zinc and insulin concentrations were plotted versus time after the dextrose challenge and the curves were fitted with spline regression using cubic splines with six knots (SAS version 9.4, SAS Institute, Cary NC, USA). The mean concentrations obtained using the two techniques were compared with a mixed effect linear model with vein and time main effects and modeling the pig as a random effect; a $p$ value of $<0.05$ was considered statistically significant. Results are summarized as mean and standard deviation unless otherwise indicated.

\section{Results}

3.1. Pancreatic Venous Drainage Sampling. It is not feasible to assess each islet that is surrounded by microcirculation in the whole pancreas. However, local whole organ venous drainage can be percutaneously sampled. In this way, the release kinetics of insulin and zinc upon glucose stimulation can be assessed with minimal influence of dilution by systemic blood. By such a sampling approach, we anticipated to determine the temporal window that allows an imaging probe for noninvasive assessment of $\beta$ cell function through zinc release upon glucose stimulation.

Prior to interventional radiology procedures, CT angiography was undertaken to obtain an idea of venous anatomy in the upper abdomen of the pig. Figure 1 is a representative illustration of the portal venous anatomy vasculature in the upper abdomen of an Ossabaw pig along with its spatial relation to the pancreatic splenic lobe (SL) and duodenal lobe (DL). The splenic vein is partially bounded by the SL of the pancreas and unites with the superior mesenteric vein to form

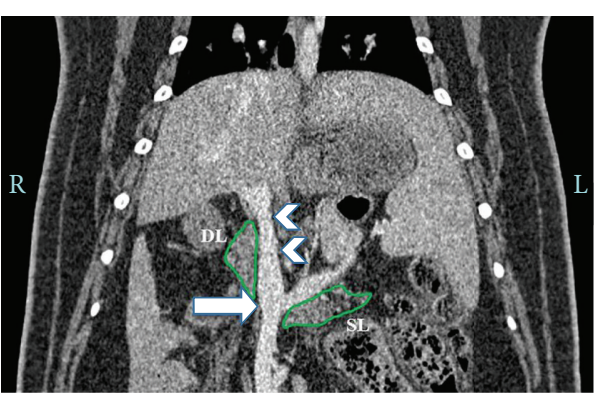

FIgure 1: Preinterventional planning CT angiography. Coronal CT angiogram demonstrating the normal vascular anatomy of the portal venous system. Note the splenoportal confluence (arrow) and the long extra-hepatic course of the portal vein (arrowheads) in relation to the pancreatic duodenal lobe (DL) and the splenic lobe (SL). Both pancreas lobes are outlined in green.

the portal vein, to which the splenic vein drains [20]. The DL of the pancreas rests against the base of the portal vein, which has a $2-3 \mathrm{~cm}$ extra-hepatic course and divides at the hepatic hilum into the right and left portal veins.

The transhepatic portal vein access was successfully performed (Figure 2). The main portal vein was accessed in 2 pigs and the right portal vein was accessed in 1 pig. Samples were obtained from the distal portal vein. The transsplenic access to the splenic vein was equally successful (Figure 3). The 2-needle technique was quicker and easier to perform. The mean (SD) time taken to access the splenic and portal veins was 34 (14) and 50 (15) min, respectively. The average number of needle passes to obtain a satisfactory access into the splenic vein after opacification was 2 . No procedural or postprocedural complications were observed in either sampling group and all pigs recovered equally well within a similar amount of time.

\subsection{Comparative Kinetics of Insulin and Zinc Release Obtained} by the Two Sampling Techniques. In the portal sampling technique, all the samples were obtained from the distal branches of the left portal vein (Figure 4(a)). Average baseline plasma zinc and insulin concentration were $1019 \pm 150 \mathrm{ppb}$ and $0.024 \pm 0.006 \mu \mathrm{g} / \mathrm{mL}$, respectively. A biphasic pattern in insulin release after the glucose challenge was noted as shown in the spline fitted results (red line tracings) (Figure 5). The first peak occurred at $2 \mathrm{~min}$ and the second was predicted to occur at $16 \mathrm{~min}$. The mean predicted concentrations were 0.10 and $0.32 \mu \mathrm{g} / \mathrm{mL}$, respectively. In the zinc release profile, there was more scatter in the data and only a single well defined peak was identified at around $1.3 \mathrm{~min}$ to a mean value of $1103 \mathrm{ppb}$.

In the splenic vein sampling technique, all samples were obtained from the splenoportal junction (Figure 4(b)). Average baseline plasma zinc and insulin concentration were $1001 \pm 298 \mathrm{ppb}$ and $0.032 \pm 0.023 \mu \mathrm{g} / \mathrm{mL}$, respectively. Samples from the splenic vein had higher insulin $(p<0.0001)$ but similar zinc concentration $(p=0.49)$ compared to the portal vein. The anticipated biphasic insulin release profile was again observed (Figure 5). The insulin concentration peaked at 2 min after the glucose challenge to a predicted value of $0.56 \pm$ 


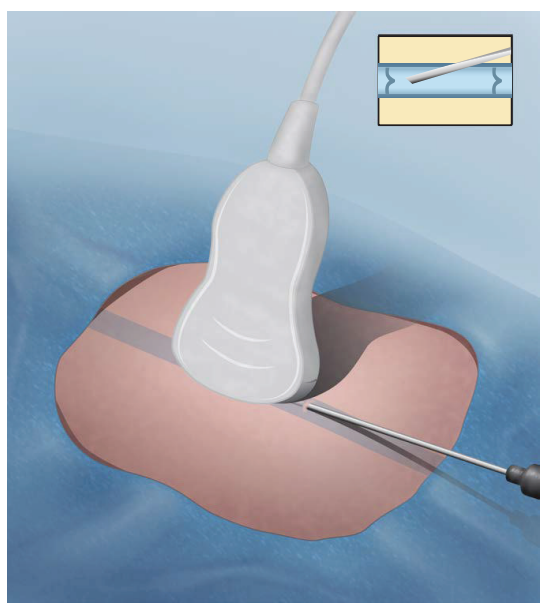

(a)

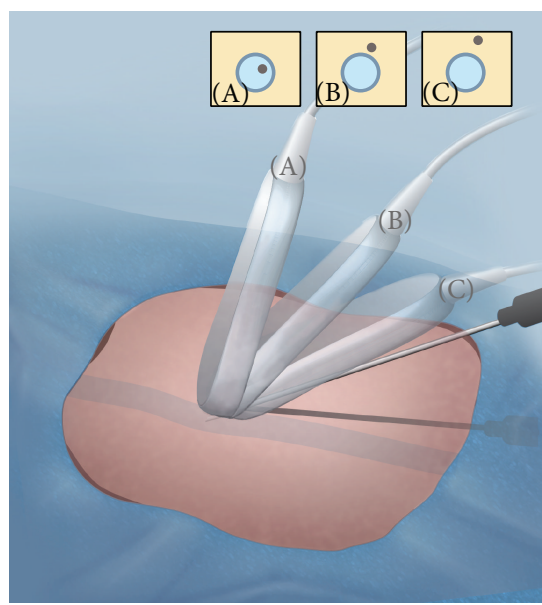

(b)

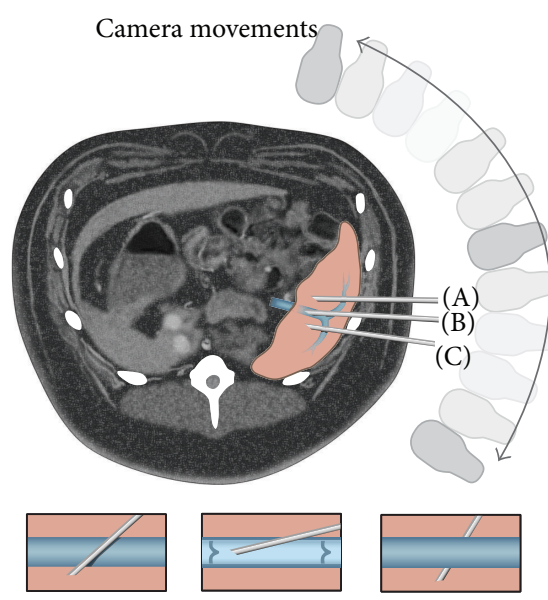

(A) Anterior
(B) In vein

(C) Posterior

(c)

FIGURE 2: Illustration of ultrasound guided venous access. (a) Illustration demonstrating the in-plane technique for the ultrasound guided access. The inset shows a schematic of the needle within the vein in long axis. (b) Illustration demonstrating out-of-plane technique for ultrasound guided access. The inset shows a schematic of the blood vessel in cross section with the dot being the needle position in the respective transducer locations. (c) Overlay illustration on an axial CT image demonstrating the relationship of the needle to the venous structure, with different positions of the fluoroscope $\mathrm{C}$ arm. The pink shading over the CT image denotes the spleen.

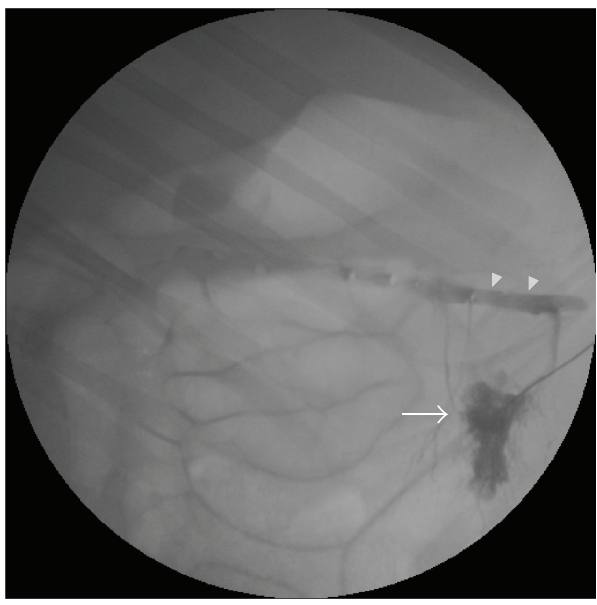

(a)

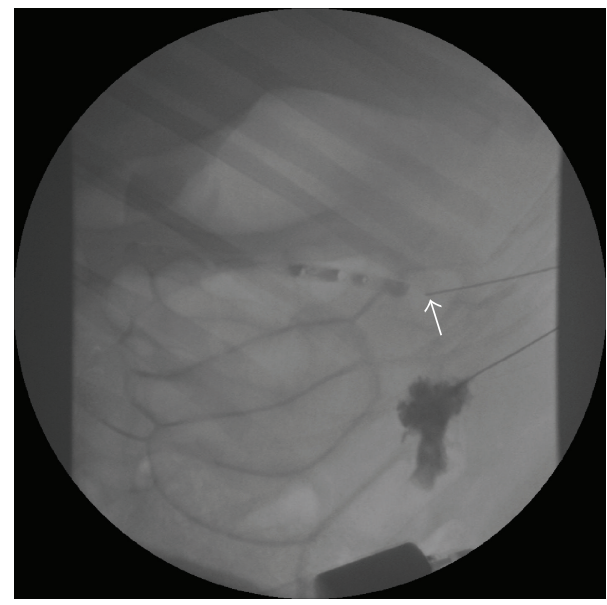

(b)

FIGURE 3: Transsplenic splenoportal venous sampling technique. (a) Digital fluoroscopic image demonstrating parenchymal blush with reflux of contrast through the splenic parenchyma opacifying the splenic vein (white arrowheads). (b) Digital fluoroscopic image demonstrating the passage of the second needle targeting the splenic vein.

$0.14 \mu \mathrm{g} / \mathrm{mL}$, an approximately 18 -fold increase of the mean. The zinc release profile again showed a single clear peak that followed the first insulin peak and was at 4 min with a mean value of $\sim 1338 \mathrm{ppb}$. The confidence interval was wider than for the portal sampling technique.

\section{Discussion}

Portal venous sampling is an essential part of pharmacological and nutritional studies. In the work presented here, portal venous sampling was utilized to find the temporal relationship of zinc release from the pancreatic $\beta$ cells upon glucose.
Portal venous access in swine was first described in 1969 by Lydtin et al. [21]. They implanted a catheter in the portal vein for repeated sampling; however, the intent was not to define the kinetics of insulin or zinc release. Over the years, many others have described techniques for portal venous sampling using various permanent implantation devices. These procedures involved open surgical techniques with associated complications of postoperative sepsis and thrombosis [22]. As demonstrated in this study, percutaneous splanchnic venous sampling is straightforward, is easily reproducible in different pigs, and can be performed efficiently and safely. While both transhepatic and transsplenic 


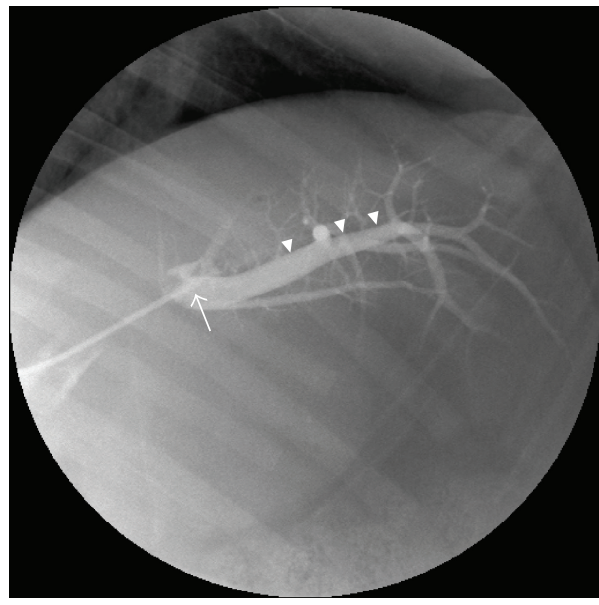

(a)

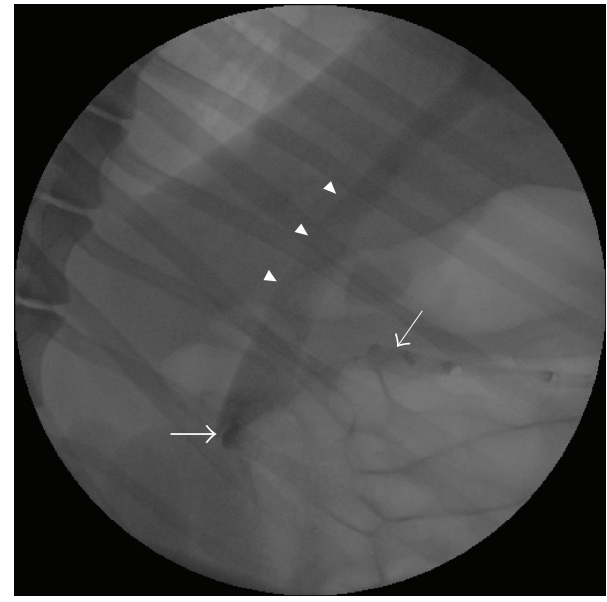

(b)

FIGURE 4: Angiographic demonstration of venous sampling sites. (a) Digital fluoroscopic image demonstrating the catheter tip (white arrow) in position for obtaining samples from the distal left portal vein (white arrowheads). (b) Digital fluoroscopic image demonstrating the splenoportal confluence (white thick arrow). Note the opacified splenic vein (white thin arrow) and the long extra-hepatic course of the portal vein (white arrowheads).

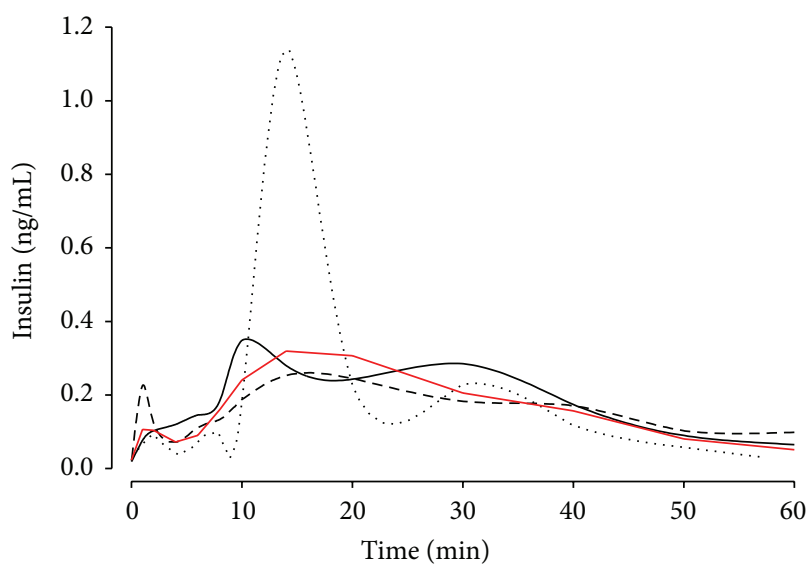

(a)

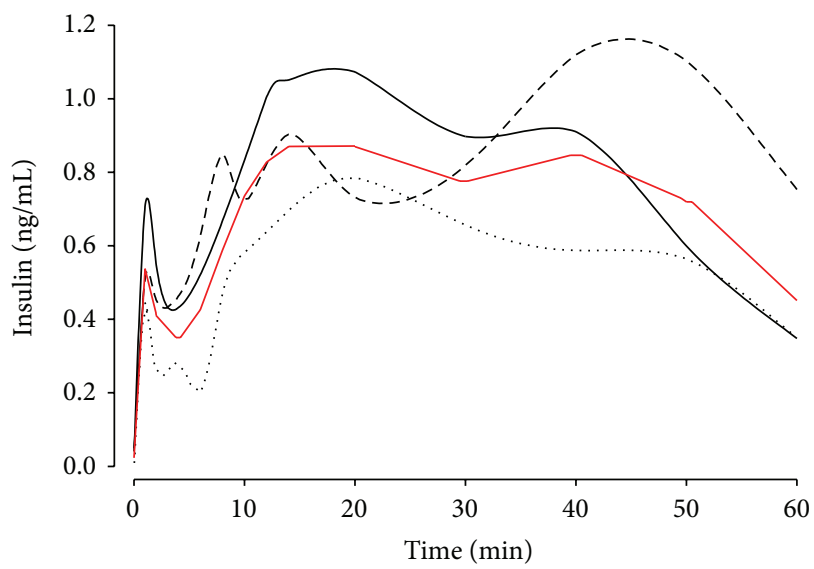

(c)

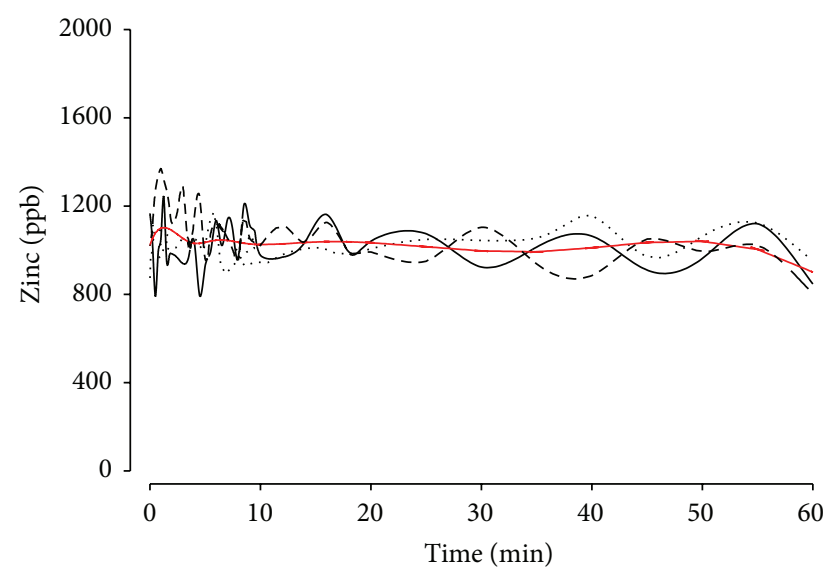

(b)

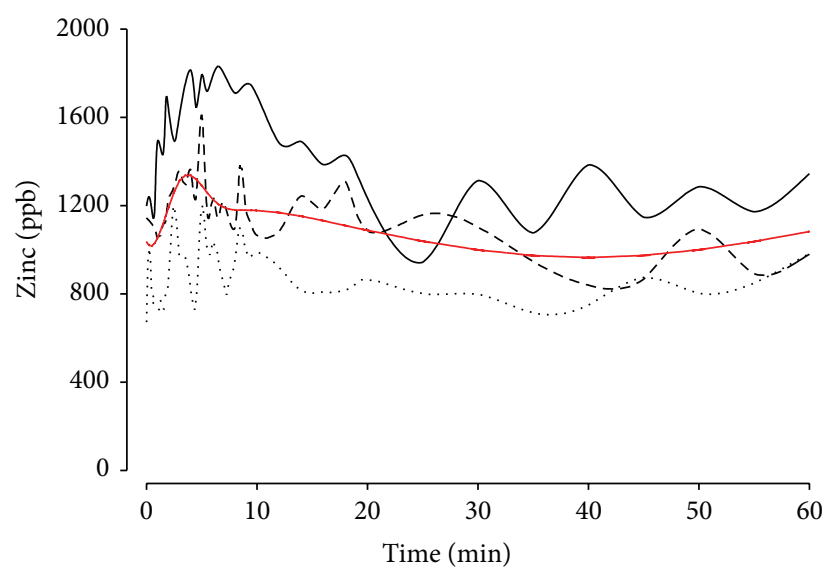

(d)

FIGURE 5: Temporal profile of insulin and zinc concentrations in the portal or splenic blood pool after injection of a dextrose bolus. Three pigs per sampling method are represented by the black dotted, dashed, and solid lines. The red curves shown are spline fits. Top row insulin (a) and zinc (b) release profiles from the transhepatic portal venous sampling technique. Bottom row insulin (c) and zinc (d) release profiles from the transhepatic splenic venous sampling technique. Note the biphasic insulin response with an associated clear rise of zinc concentration after the first peak at approximately 5 minutes. 
image-guided techniques were reproducible and safe, sampling from the splenic vein via transsplenic access took less time and was technically easier to perform.

It is known that insulin release from the pancreatic $\beta$ cell after glucose challenge is biphasic. The first phase of the insulin response is the result of release of preformed granules. This phase is almost invariably reduced in patients with impaired glucose tolerance test or in early stages of type 2 diabetes before overt symptoms of the disease manifest [23]. In our study, we observed a biphasic pattern of insulin release using either the transhepatic technique with portal venous sampling or the transsplenic technique with splenic sampling. Additionally, the splenic vein samples had higher mean concentration peak values for both insulin and zinc. This observation possibly resulted from dilution by the mixing with blood from the superior mesenteric vein. Sampling without mesenteric venous contamination can also be achieved from the portal venous access by placing a catheter under fluoroscopic guidance into the splenoportal confluence from the portal access site. The fact that the distal portal vein radicles are smaller than the splenoportal confluence might also contribute to the sampling errors and also explain the higher concentration of insulin and zinc in the splenic vein cohort. The temporal secretory correlation between insulin and zinc was inconsistent in both cohorts. The correlation was numerically, but not statistically, higher in the splenic vein cohort. The explanation of the inconsistency is presently unclear but may reflect a physiological range in insulin and zinc correlations in pigs. Nevertheless, we achieved one of our aims which was to define a time interval for administration of zinc targeting radiotracer imaging after a glucose challenge. Based on our results, an optimal window for injection of a tracer targeting zinc release would be in the $2-5$ min time frame.

Our study has some limitations. Firstly, initially there were animal welfare concerns that caused us to perform the two techniques on different pigs. Based on our experience in this study, a pig could undergo venous sampling by both techniques. An added benefit of this study design could be mitigation of variation caused by physiological differences in animals. Secondly, the sample sizes in each group were small.

In summary, our study assessed the temporal pattern of insulin and zinc release after glucose challenge using 2 different techniques for venous sampling. These techniques use small catheters, which are not traumatic and can be removed after the sampling is performed without any complications. It should be possible to safely perform repeated access in the same animal. Based on the data from two cohorts of Ossabaw minipigs, we conclude that the splenic vein access for sampling is technically easier and superior to transhepatic portal vein access for studying $\beta$ cell function after glucose challenge. To our knowledge, neither the biphasic insulin release profile nor the temporal profile of zinc release in response to a glucose challenge has been reported in swine as demonstrated here. The splenoportal venous sampling via the transsplenic access technique presented herein may find applications in testing the efficacy of therapeutics aimed at improving $\beta$ cell function.

\section{Disclosure}

This paper was previously presented as an educational exhibit at the swine in biomedical research conference 2014 at Raleigh, North Carolina, July 6-8, 2014.

\section{Conflict of Interests}

The authors declare that there is no conflict of interests regarding the publication of this paper.

\section{Acknowledgments}

The authors would like to acknowledge Townsend Majors and Pam Curry for the illustrations and Susan Miller, Brian Gutowski, and Angela Guillory for technical assistance. This paper was funded by Juvenile Diabetes Research Foundation International (JDRFI-37-2011-632-02), National Institute of Health (R01 DK092163).

\section{References}

[1] D. Li, S. Chen, E. A. Bellomo et al., "Imaging dynamic insulin release using a fluorescent zinc indicator for monitoring induced exocytotic release (ZIMIR)," Proceedings of the National Academy of Sciences of the United States of America, vol. 108, no. 52, pp. 21063-21068, 2011.

[2] National Center for Health Statistics, Healthy People 2010: Final Review, vol. 1 of DHHS Publication, US Department of Health and Human Services, Centers for Disease Control and Prevention, National Center for Health Statistics, Hyattsville, Md, USA, 2012.

[3] A. J. M. Lubag, L. M. De Leon-Rodriguez, S. C. Burgess, and A. D. Sherry, "Noninvasive MRI of $\beta$-cell function using a $\mathrm{Zn}^{2+}$ responsive contrast agent," Proceedings of the National Academy of Sciences of the United States of America, vol. 108, no. 45, pp. 18400-18405, 2011.

[4] A. C. Esqueda, J. A. López, G. Andreu-de-Riquer et al., "A new gadolinium-based MRI zinc sensor," Journal of the American Chemical Society, vol. 131, no. 32, pp. 11387-11391, 2009.

[5] K. Lemaire, M. A. Ravier, A. Schraenen et al., "Insulin crystallization depends on zinc transporter ZnT8 expression, but is not required for normal glucose homeostasis in mice," Proceedings of the National Academy of Sciences of the United States of America, vol. 106, no. 35, pp. 14872-14877, 2009.

[6] K. G. Slepchenko, C. B. L. James, and Y. V. Li, "Inhibitory effect of zinc on glucose-stimulated zinc/insulin secretion in an insulin-secreting $\beta$-cell line," Experimental Physiology, vol. 98, no. 8, pp. 1301-1311, 2013.

[7] T. V. O’Halloran, M. Kebede, S. J. Philips, and A. D. Attie, “Zinc, insulin, and the liver: a ménage à trois," The Journal of Clinical Investigation, vol. 123, no. 10, pp. 4136-4139, 2013.

[8] T. J. Nicolson, E. A. Bellomo, N. Wijesekara et al., "Insulin storage and glucose homeostasis in mice null for the granule zinc transporter ZnT8 and studies of the type 2 diabetesassociated variants," Diabetes, vol. 58, no. 9, pp. 2070-2083, 2009.

[9] L. D. Pound, S. A. Sarkar, R. K. P. Benninger et al., "Deletion of the mouse Slc30a8 gene encoding zinc transporter-8 results in impaired insulin secretion," Biochemical Journal, vol. 421, no. 3, pp. 371-376, 2009. 
[10] N. Wijesekara, F. F. Dai, A. B. Hardy et al., "Beta cell-specific Znt8 deletion in mice causes marked defects in insulin processing, crystallisation and secretion," Diabetologia, vol. 53, no. 8, pp. 1656-1668, 2010.

[11] A. Caumo and L. Luzi, "First-phase insulin secretion: does it exist in real life? Considerations on shape and function," The American Journal of Physiology: Endocrinology and Metabolism, vol. 287, no. 3, pp. E371-E385, 2004.

[12] R. E. Pratley and C. Weyer, "The role of impaired early insulin secretion in the pathogenesis of Type II diabetes mellitus," Diabetologia, vol. 44, no. 8, pp. 929-945, 2001.

[13] D. J. Michael, R. A. Ritzel, L. Haataja, and R. H. Chow, "Pancreatic $\beta$-cells secrete insulin in fast- and slow-release forms," Diabetes, vol. 55, no. 3, pp. 600-607, 2006.

[14] O. L. Munk, S. Keiding, and L. Bass, "Impulse-response function of splanchnic circulation with model-independent constraints: theory and experimental validation," The American Journal of Physiology_Gastrointestinal and Liver Physiology, vol. 285, no. 4, pp. G671-G680, 2003.

[15] G. M. Kaiser, N. R. Frühauf, and C. E. Broelsch, "New surgical technique for portal venous port system in swine," European Surgical Research, vol. 37, no. 5, pp. 298-301, 2005.

[16] F. G. Court, S. A. Wemyss-Holden, C. P. Morrison et al., "Segmental nature of the porcine liver and its potential as a model for experimental partial hepatectomy," British Journal of Surgery, vol. 90, no. 4, pp. 440-444, 2003.

[17] L. Lee, M. Alloosh, R. Saxena et al., "Nutritional model of steatohepatitis and metabolic syndrome in the Ossabaw miniature swine," Hepatology, vol. 50, no. 1, pp. 56-67, 2009.

[18] P. Garcia, G. Genin, P. M. Bret, V. M. Bonaldi, C. Reinhold, and M. Atri, "Hepatic CT enhancement: effect of the rate and volume of contrast medium injection in an animal model," Abdominal Imaging, vol. 24, no. 6, pp. 597-603, 1999.

[19] N. L. Miller, B. R. Matlaga, and J. E. Lingeman, “Techniques for fluoroscopic percutaneous renal access," Journal of Urology, vol. 178, no. 1, pp. 15-23, 2007.

[20] J. Ferrer, W. E. Scott III, B. P. Weegman et al., "Pig pancreas anatomy: implications for pancreas procurement, preservation, and islet isolation," Transplantation, vol. 86, no. 11, pp. 15031510, 2008.

[21] H. Lydtin, M. Pieper, T. Kusus, K. Schnelle, and N. Zöllner, "Chronic implantation of catheters into the portal vein, the inferior vena cava, the right atrium and the stomach of the miniature pig," Zeitschrift für Die Gesamte Experimentelle Medizin, vol. 149, no. 3, pp. 279-282, 1969.

[22] M. B. Bailie, S. K. Wixson, and M. S. Landi, "Vascular-accessport implantation for serial blood sampling in conscious swine," Laboratory Animal Science, vol. 36, no. 4, pp. 431-433, 1986.

[23] A. Caumo and L. Luzi, "First-phase insulin secretion: does it exist in real life? Considerations on shape and function," The American Journal of Physiology -Endocrinology \& Metabolism, vol. 287, no. 3, pp. E371-E385, 2004. 


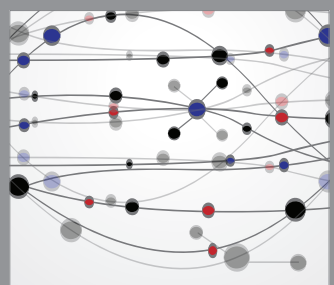

The Scientific World Journal
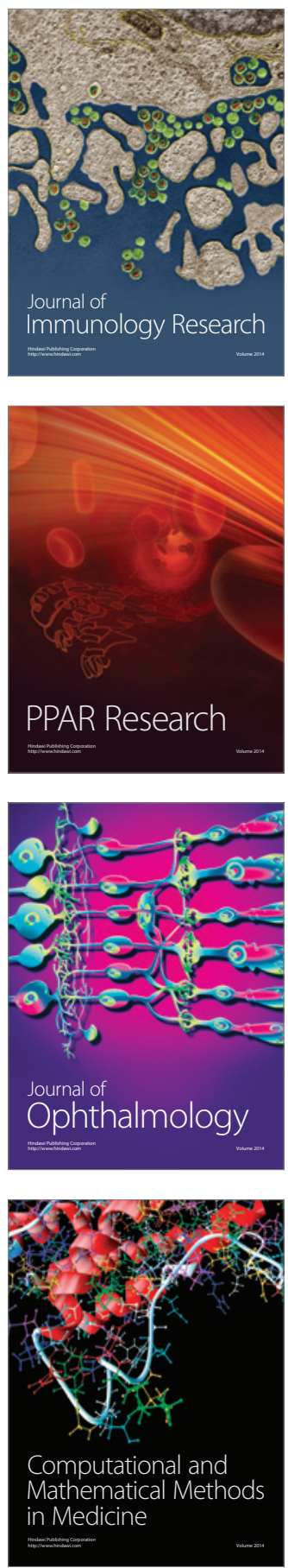

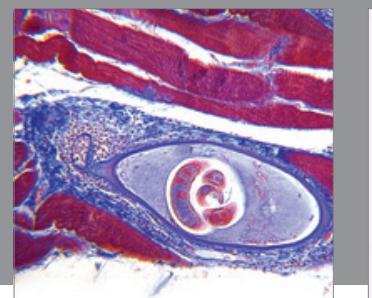

Gastroenterology

Research and Practice
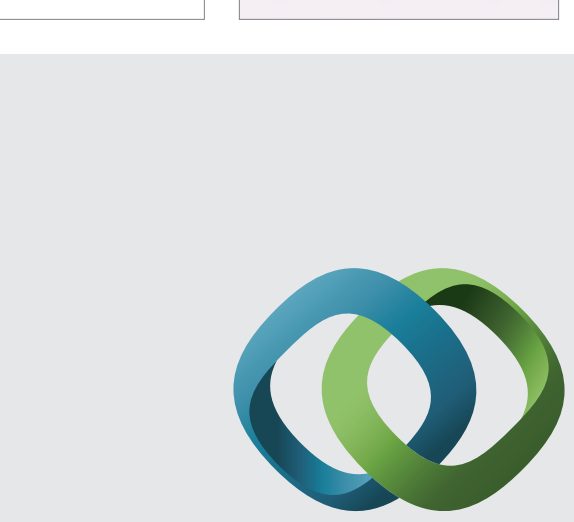

\section{Hindawi}

Submit your manuscripts at

http://www.hindawi.com
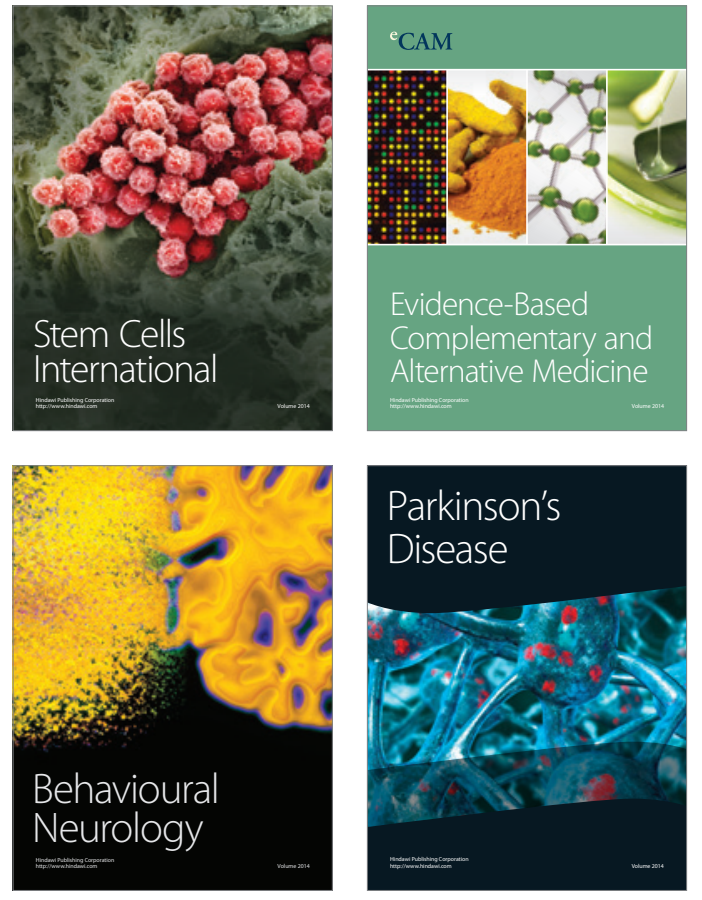
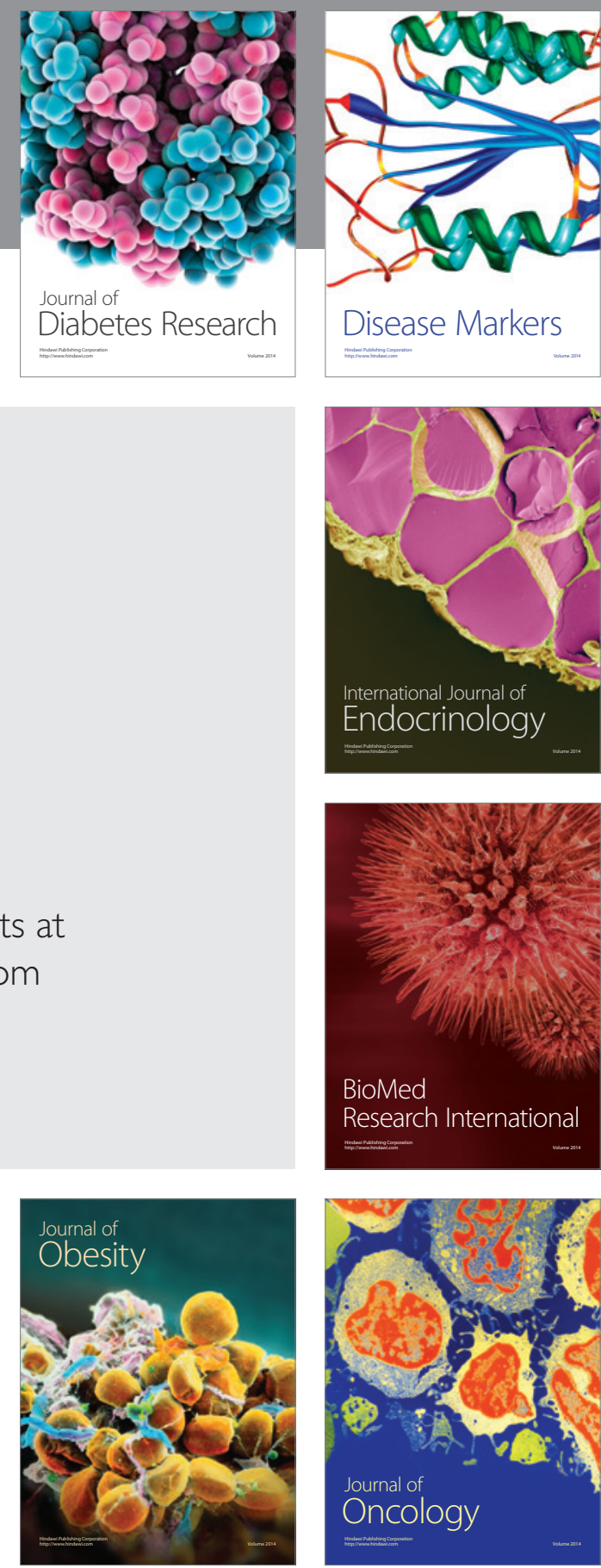

Disease Markers
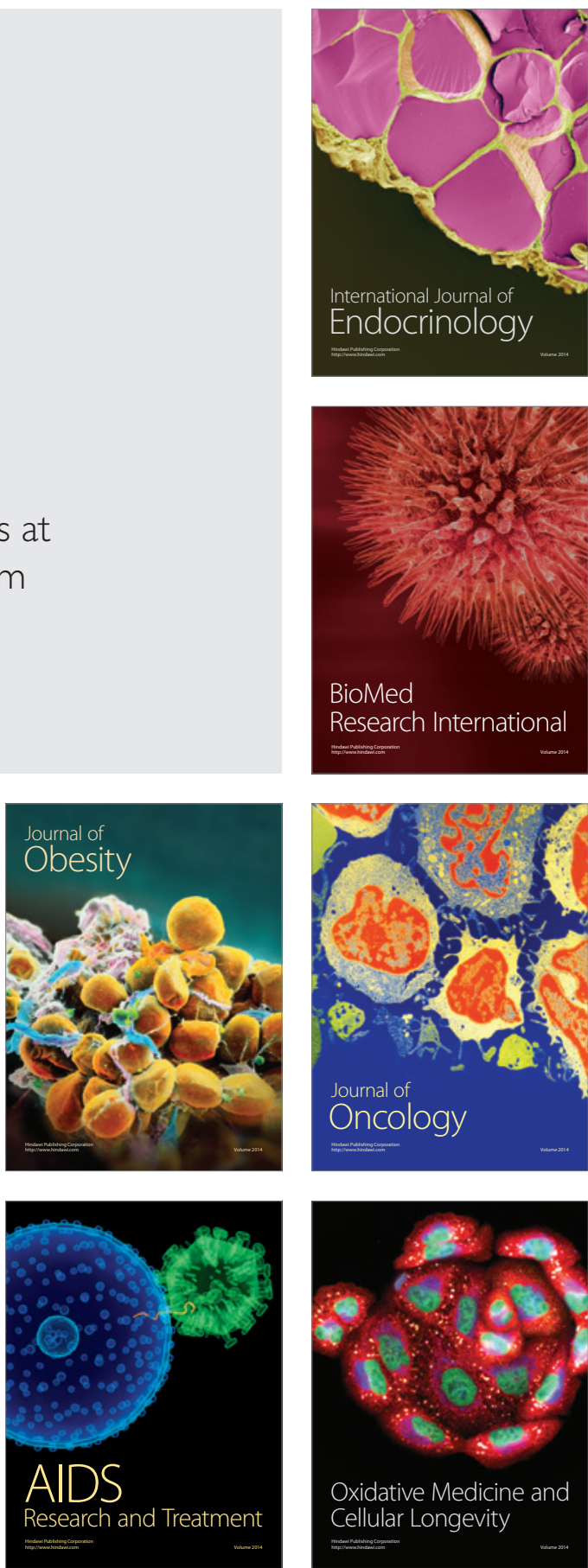